\title{
ANALYTIC EQUATIONS AND SINGULARITIES OF PLANE CURVES
}

BY

JOHN J. WAVRIK

\begin{abstract}
Theorems (Artin, Wavrik) exist which show that sufficiently good approximate (power series) solutions to a system of analytic equations may be approximated by convergent solutions. This paper considers the problem of explicity determining the order, $\beta$, to which an approximate solution must solve the system of equations.

The paper deals with the case of one equation, $f(x, y)=0$, in two variables. It is shown how $\beta$ depends on the singularities of the curve $f(x, y)=0$. A method for obtaining the minimal $\beta$ is given. A rapid way of finding $\beta$ using the Newton Polygon for $f$ applies in special cases.
\end{abstract}

Introduction. Let $k$ be a field of characteristic 0 complete with respect to a nontrivial valuation. We use $k\{\}$ to denote convergent power series; $k[[]]$ to denote formal power series; and $k$ [ ] to denote polynomials. Let $x=$ $\left(x_{1}, \ldots, x_{n}\right), y=\left(y_{1}, \ldots, y_{p}\right), z=\left(z_{1}, \ldots, z_{r}\right), f=\left(f_{1}, \ldots, f_{m}\right)$ with $f_{i} \in$ $k\{x, y\}[z]$.

The following theorem was proved in [7]:

TheOREM. $\forall \alpha \exists \beta$ such that if $\bar{y}(x) \in k[[x]]^{p}$ with $\bar{y}(0)=0$ and $\bar{z}(x) \in$ $k[[x]]^{r}$ satisfy

$$
f(x, \bar{y}(x), \bar{z}(x)) \equiv 0 \bmod (x)^{\beta^{\prime}} .
$$

Then $\exists y(x) \in k\{x\}^{p}$ with $y(0)=0$ and $z(x) \in k\{x\}^{r}$ such that

(1) $f(x, y(x), z(x))=0$,

(2) $y(x) \equiv \bar{y}(x), z(x) \equiv \bar{z}(x) \bmod (x)^{\alpha}$.

This result may be paraphrased by saying that sufficiently good approximate solutions to a system of analytic equations can be approximated by actual solutions. The corresponding theorem in the case in which the $f_{i}$ are polynomials was obtained by Artin in [1]. The theorem has applications in deformation theory (see [7])-it is the genesis of results asserting the existence of only finitely many obstructions to deformation. The proof given in [7] is

Received by the editors August 11, 1977 and, in revised form, November 11, 1977.

AMS (MOS) subject classifications (1970). Primary 32B99, 30A10, 65H05; Secondary 32C40, $14 \mathrm{H} 20$.

Key words and phrases. Analytic equations, plane curve singularities, power series, algebraic functions.

() American Mathematical Society 1979 
purely existential. No calculation, estimate, or bound for $\beta$ is obtained from the proof. If the theorem is to be applied to a deformation theory problem, no means is provided for determining when the last obstruction is encountered. The purpose of the present paper is to explore means for explicitly calculating $\beta$.

The number $\beta$ must depend on the nature of the singularity determined by $f(x, y, z)=0$. To clarify this connection we restrict to the case $n=p=m=$ $1, r=0, f$ a polynomial. Thus we seek to find solutions of a single polynomial equation, $f(x, y)=0$, in two variables.

The proof in [7] uses a complex multiple induction. An approach to the calculation of $\beta$ would be a more detailed analysis of that proof. In $\$ 1$ we present a drastically simplified version of that proof. This simplified proof yields an explicit value for $\beta$ which, unfortunately, is not usually close to the minimal value.

In $\$ 2$ an alternative proof is provided which is based on the resolution of singularities by quadratic transformations. This proof provides a criterion for determining when the last obstruction is encountered.

In $\$ 3$ the resolution scheme is used to find the minimum $\beta$ in specific cases.

In $\$ 4$ a method for rapidly determining $\beta$ from the Newton Polygon for $f$ is discussed.

\section{A proof of the theorem.}

THEOREM I. Given $\alpha>0$ and $f(x, y) \in k[x, y] \exists \beta$ such that if $\bar{y}(x) \in k[[x]]$ satisfies $f(x, \bar{y}(x)) \equiv 0 \bmod (x)^{\beta}$ then $\exists y(x) \in k[[x]]$ with

(1) $f(x, y(x))=0$,

(2) $y(x) \equiv \bar{y}(x) \bmod (x)^{\alpha}$.

THEOREM II. Given $f, g \in k[x, y] \forall \gamma \exists \beta$ such that if $\bar{y}(x) \in k[[x]]$ satisfies

(i) $f(x, \bar{y}(x)) \equiv 0 \bmod \left(g(x, \bar{y}(x))+(x)^{\beta}\right)$,

(ii) $g(x, \bar{y}(x)) \neq 00 \bmod (x)^{\gamma}$, then $f(x, \bar{y}(x)) \equiv 0 \bmod (g(x, \bar{y}(x)))$.

Proof OF THEOREM II. We show that $\beta=\gamma-1$ satisfies the requirements. Let $\bar{y}(x)$ satisfy the hypothesis. If $g(x, \bar{y}(x))$ is a unit in $k[[x]]$ then the conclusion is obvious. If $g(x, \bar{y}(x))$ is not a unit then we write $g(x, \bar{y}(x))=$ $x^{s} u(x)$ where $u(0) \neq 0$ and $1 \leqslant s<\gamma$.

Now

$$
\begin{aligned}
f(x, \bar{y}(x)) & =A(x) g(x, \bar{y}(x))+B(x) x^{\beta} \\
& =\left(A(x)+(u(x))^{-1} B(x) x^{\beta-s}\right) g(x, \bar{y}(x)) \\
& \equiv 0 \bmod (g(x, \bar{y}(x))),
\end{aligned}
$$

since $\beta=\gamma-1 \geqslant s$. 
Proposition 1. Given $f(x, y) \in k[x, y] \forall \alpha, \gamma \exists \beta \geqslant \gamma$ such that if $\bar{y}(x) \in$ $k[[x]]$ satisfies

$$
\begin{aligned}
f(x, \bar{y}(x)) \equiv 0 & \bmod (x)^{\beta} \\
f_{y}(x, \bar{y}(x)) \neq 0 & \bmod (x)^{\gamma}
\end{aligned}
$$

then $\exists y(x) \in k[[x]]$ with

(1) $f(x, y(x))=0$,

(2) $y(x) \equiv \bar{y}(x) \bmod (x)^{\alpha}$.

Proof. Let $g=\left(f_{y}(x, y)\right)^{2}$. We will show that $\beta=\alpha+2 \gamma$ satisfies the requirements.

Since $f(x, \bar{y}(x)) \equiv 0 \bmod (x)^{\beta}$ we have $f(x, \bar{y}(x)) \equiv 0 \bmod (g(x, \bar{y}(x))+$ $\left.(x)^{\beta}\right)$. Also $\beta=\alpha+2 \gamma \geqslant 2 \gamma-1$ and $g(x, \bar{y}(x)) \neq 0 \bmod (x)^{2 \gamma}$. By the previous theorem we see that $f(x, \bar{y}(x)) \equiv 0 \bmod (g(x, \bar{y}(x)))$.

Let $F(x, Y)=f(x, \bar{y}(x)+Y)$ so that $F(x, 0)=f(x, \bar{y}(x))$ and

$$
\partial F(x, 0) / \partial Y=f_{y}(x, \bar{y}(x)) \text {. }
$$

Let $J$ be the ideal in $k[[x]]$ generated by $\partial F(x, 0) / \partial Y$ and $J^{\prime}=(x)^{\alpha}$. We have $f(x, \bar{y}(x))=B(x)\left(f_{y}(x, \bar{y}(x))\right)^{2}$. The left side is $\equiv 0 \bmod (x)^{\beta}$ while $\left(f_{y}(x, \bar{y}(x))\right)^{2} \neq 0 \bmod (x)^{2 \gamma}$ thus $B(x) \equiv 0 \bmod (x)^{t}$ with $t \geqslant \beta-2 \gamma=\alpha$. This shows that $F(x, 0) \in J^{2} \cdot J^{\prime}$. We now apply the Tougeron Implicit Function Theorem ([5, III.3.2], or [7, Theorem 3]): there is a $\hat{y}(x) \in J \cdot J^{\prime}$ such that $F(x, \hat{y}(x))=0$. Set $y(x)=\bar{y}(x)+\hat{y}(x)$. Then $f(x, y(x))=0$ and $y(x) \equiv \bar{y}(x) \bmod (x)^{\alpha}$ as required.

Proof of Theorem I. The task is to reduce the proof to a case of Proposition 1.

Write $f(x, y)$ and $f_{y}(x, y)$ in powers of $y$. Let $D(x)$ denote the discriminant of $f$ (as a polynomial in $y$ ). We can find [6, Theorem 9.6] polynomials $A(x, y)$, $B(x, y) \in k[x, y]$ so that

$$
D(x)=A(x, y) f(x, y)+B(x, y) f_{y}(x, y) .
$$

Case 1. $f$ is irreducible.

In this case $D(x) \neq 0$. Let $d=\operatorname{ord}(D(x))$. If $\bar{y}(x) \in k[[x]]$ satisfies $f(x, \bar{y}(x)) \equiv 0 \bmod (x)^{d}$ then we see from $(*)$ that $f_{y}(x, \bar{y}(x)) \neq 0 \bmod (x)^{d}$. Let $\alpha$ be given and set $\beta=2 d+\alpha$. By Proposition 1 we find a solution, $y(x)$, to $f(x, y(x))=0$ such that $y(x) \equiv \bar{y}(x) \bmod (x)^{\alpha}$.

Case 2. $f$ is reducible.

Let $f=f_{1} \cdots f_{t}$ be a factorization of $f$ into irreducible factors. Let $\beta_{i}$ satisfy the condition of Theorem I for the equation $f_{i}(x, y)=0$. Let $\beta=\beta_{1}$ $+\cdots+\beta_{t}$. If $f(x, \bar{y}(x)) \equiv 0 \bmod (x)^{\beta}$ then $\exists i$ s.t. $f_{i}(x, \bar{y}(x)) \equiv 0 \bmod (x)^{\beta_{i}}$. By Theorem I, applied to $f_{i}$, we find $y(x)$ such that $f_{i}(x, y(x))=0$ hence also $f(x, y(x))=0$. Moreover $y(x) \equiv \bar{y}(x) \bmod (x)^{\alpha}$. 
Notice that we have not only proved the theorem, but we even have a formula for $\beta$ that satisfies the conditions:

If $f(x, y) \in k[x, y]$ is irreducible and $d=\operatorname{ord}(D(x))$, where $D(x)$ is the discriminant of $f$, then $\beta=2 d+\alpha$ satisfies the conditions of Theorem I. If $f(x, y)$ factors, $f=f_{1} \cdots f_{t}$ with $f_{i}$ irreducible, then $\beta=\beta_{1}+\cdots+\beta_{t}$ with $\beta_{i}$ as above for $f_{i}$.

2. $\beta$ and the resolution of singularities. In this section an outline is given of an alternative proof of Theorem I.

We will always assume that $f(x, y)=0$ has no multiple solutions. This will be the case, for example, when $f(x, y)$ is irreducible.

Proposition 2. If $f(x, y)=0$ is regular at $\left(0, a_{0}\right)$ then there is a solution to $f(x, y)=0$ with $y(0)=a_{0}$ if and only if there is a solution to $f(x, y) \equiv 0 \mathrm{mod}$ $(x)^{2}$ with $y(0)=a_{0}$.

Proof. Apply the Implicit Function Theorem.

We will refer to this as the "IFT case".

Remarks. (1) In the IFT case, $\beta=\max (\alpha, 2)$ satisfies the conditions of Theorem I.

(2) The solution is unique in this case and the $a_{i}(i \geqslant 1)$ can be calculated by recursion.

(3) Kung and Traub [4] provide an algorithm for computing the $a_{i}(i>1)$ with lower "cost" by using Newton Iteration.

Now suppose that $f(x, y)=0$ has an $r_{0}$-fold point at $\left(0, a_{0}\right)$ with $r_{0}>1$. We write $f\left(x, a_{0}+x y\right)=x^{r_{0}} f_{1}(x, y)$.

DEFINITION 1. $f_{1}(x, y)=0$ is called the proper transform of $f(x, y)=0$ under the standard quadratic transformation with center $\left(0, a_{0}\right)$.

Definition 2. The points $\left(0, a_{1}\right)$ satisfying $f_{1}\left(0, a_{1}\right)=0$ are said to lie over $\left(0, a_{0}\right)$.

The process is repeated using $f_{1}$ and the $\left(0, a_{1}\right)$.

Proposition 3. A plane curve singularity may be resolved by a finite sequence of standard quadratic transformations.

Remark. The proof of Proposition 3 is found in [3]. Bounds for the number of quadratic transformations required can be given in terms of the "apparent genus" of the curve and, ultimately, in terms of the degree of $f(x, y)$.

Proposition 4. If $y=a_{0}+a_{1} x+\cdots+a_{k} x^{k}$ satisfies $f(x, y) \equiv 0 \mathrm{mod}$ $(x)^{\beta}$ for $\beta$ sufficiently large, then the $\left(0, a_{i}\right)$ are centers of successive quadratic transformations and conversely.

Proof. If $\left(0, a_{0}\right)$ is an $r_{0}$-fold point then $y=a_{0}+a_{1} x+\ldots$ satisfies $f(x, y) \equiv 0 \bmod (x)^{\beta}$ if and only if $y_{1}=a_{1}+a_{2} x+\ldots$ satisfies $f_{1}\left(x, y_{1}\right) \equiv$ 
$0 \bmod (x)^{\beta-r_{0}}$. The proof follows from this.

REMARKs. (1) Since a finite (bounded in advance) sequence of quadratic transformations leads to regular points, the IFT case will apply after a finite number of steps.

(2) The last obstruction is encountered when $f_{k}(x, y)=0$ is regular at $\left(0, a_{k}\right)$.

(3) Theorem I follows from Propositions 3 and 4.

(4) Since the multiplicity of a singularity does not increase under quadratic transformation, a bound for $\beta$ can be obtained. This bound is usually excessively large.

3. Computing the minimum $\beta$. We record the results of the resolution process of $\$ 2$ in the form of a "tree diagram" showing the points that arise in successive quadratic transformations and their multiplicities. This diagram is similar to the classical diagram of "infinitely near points" to a singularity.

EXAMPLE 1. $f(x, y)=y^{5}+2 x y^{4}-x y^{2}-2 x^{2} y+\left(x^{4}-x^{3}\right)$ [6, Chapter IV, §3.3] has the tree diagram ${ }^{3}$ at $(0,0)$. Thus $(0,0)$ is a 3 -fold point with a simple point lying over it. The termination of the diagram means that the simple point has no points lying over it (it has a vertical tangent!).

EXAMPLE 2. $f(x, y)=y^{4}-y^{3}-2 x y^{2}+\left(2 x^{4}-x^{2}\right) y+x^{5}$ (this is the preceding example with $x$ and $y$ interchanged) has tree diagram

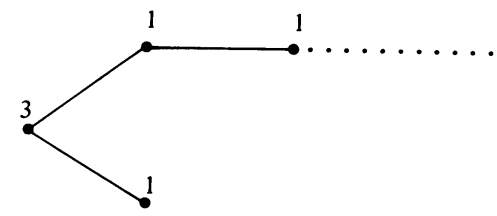

at $(0,0) .(0,0)$ is a triple point with two simple points lying over it. One of the simple points has no points over it ... the other has a sequence of simple points over it.

The tree diagram will always have a finite number of branches some of which terminate and some which continue indefinitely with a line of simple points. We attach to the $i$ th branch in the tree the number $\beta_{i}=\beta_{i}(\alpha)$.

Definition 3. (a) For a terminating branch with multiplicities $r_{0}, \ldots, r_{k}$ set $\beta_{i}=r_{0}+\cdots+r_{k}+1$.

(b) For a nonterminating branch with multiplicities $r_{i}$ set $\beta_{i}=\sum_{i=0}^{\alpha-2} r_{i}+1$.

THEOREM 1. $\beta=\max \left(\beta_{i}\right)$ is the minimum $\beta$ which satisfies the conditions of Theorem I.

Proof. This follows from the observation used in the proof of Proposition 4.

REMARK. For a nonterminating branch there is a smallest $k$ so that $r_{i}=1$ 
when $i \geqslant k$. If $\alpha>k$ we obtain $\beta_{i}=r_{0}+\cdots+r_{k-1}-k+\alpha$. We can always use this procedure for the case of a nonterminating branch: Take the sum of the multiplicities of all vertices corresponding to singular points, subtract the number of such vertices and add $\alpha$.

EXAMPLES. (1) $f(x, y)=y^{5}+2 x y^{4}-x y^{2}-2 x^{2} y+\left(x^{4}-x^{3}\right)$. $\beta=5$.

(2) $f(x, y)=y^{4}-y^{3}-2 x y^{2}+\left(2 x^{4}-x^{2}\right) y+x^{5} \cdot \beta=\max (5, \alpha+2)$.

(3) $f(x, y)=y^{5}-x^{2} y^{2}+x^{5}[2$, p. 48, Example 2]. $\beta=6$.

(4) $f(x, y)=y^{n}-x^{m}(n, m>0, n \nmid m) . \beta=m+1$. (We may compare this with the value $\beta=2 m(n-1)+\alpha$ found in $\S 1$.)

(5) $f(x, y)=y^{n}-x^{k n}(n, k>0) . \beta=k n-k+\alpha$ (minimum if $\left.\alpha>k\right)$.

4. $\beta$ and the Newton Polygon. In this section we show how $\beta$ may easily be obtained from the Newton Polygon for $f(x, y)$ in special cases.

These methods apply only to solutions that begin with $a_{0}=0$. For $a_{0} \neq 0$ the origin of the coordinate system must be translated.

We will also assume that $y$ is not a factor of $f(x, y)$. The case in which it is a factor is treated at the end of the section.

Let $f(x, y)=\sum a_{i j} x^{i} y^{j}$. Let $I=\left\{(i, j) \mid a_{i j} \neq 0\right\}$. We define a partial order on $\mathbf{R}^{2}$ by $\left(x_{1}, y_{1}\right) \leqslant\left(x_{2}, y_{2}\right)$ if $x_{1} \leqslant x_{2}$ and $y_{1} \leqslant y_{2}$. Let $S=\left\{(x, y) \in \mathbf{R}^{2} \mid(x, y)\right.$ $\geqslant(i, j)$ for some $(i, j) \in I\}$ and let $\hat{S}$ be the convex closure of $S$.

Definition 4. The Newton Polygon of $f$ at $(0,0)$ is that part of the boundary of $\hat{S}$ which is not contained in the coordinate axes.

Examples. The Newton Polygons for Examples 1-5 are given in Figures $1-4$.

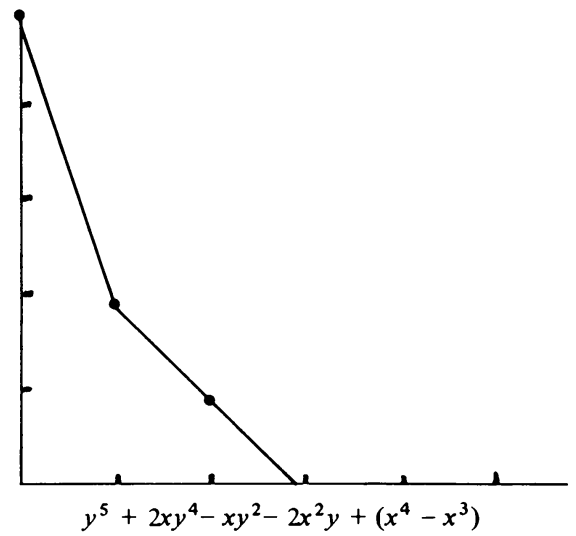

FIGURE 1

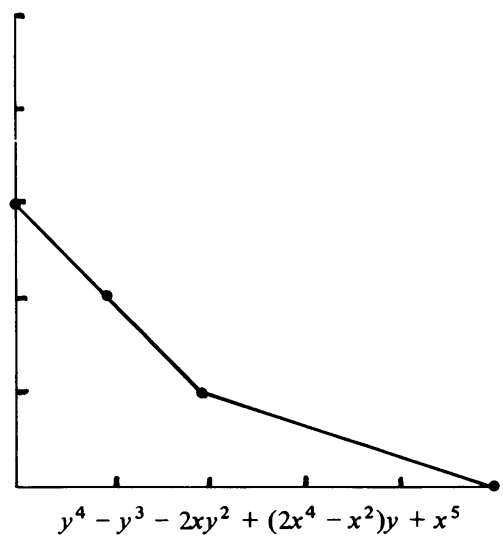

FIGURE 2 


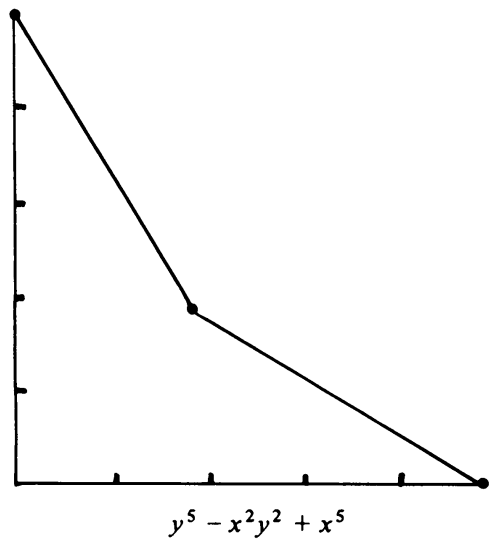

FIGURE 3

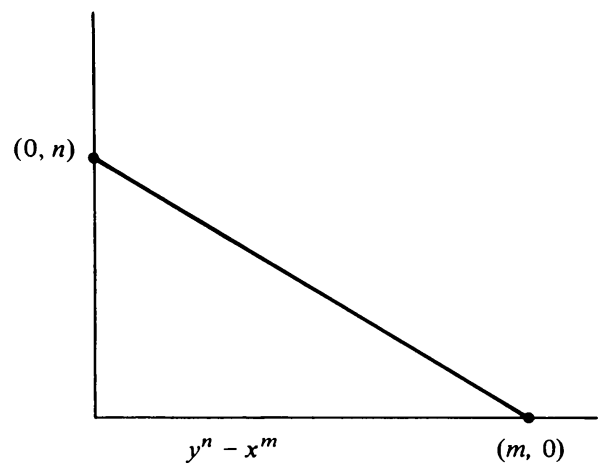

FIGURE 4

Definition 5. By the declivity of a segment of the Newton Polygon we mean the negative of the reciprocal of its slope.

Thus if $d$ is the declivity the segment is part of a line whose equation is $x+d y=\lambda$ for some constant $\lambda$.

Definition 6. A Newton Polygon is called simple if every segment of integral declivity contains only two vertices.

The Newton Polygons in Figures 3 and 4 are simple. The Newton Polygons of Figures 1 and 2 are not simple.

Let $m=\operatorname{ord}(f(x, 0))$. If $x+d y=\lambda$ for $d$ and $\lambda$ integral is the equation of a segment of the Newton Polygon we put $\lambda_{d}=\lambda$.

Theorem 2. If the Newton Polygon for $f(x, y)$ is simple then $\beta=\max (m+$ $\left.1, \lambda_{d}-d+\alpha\right)$ satisfies the conditions of Theorem $\mathrm{I}$.

Proof. $\bar{y}(x) \equiv 0$ can solve $f(x, \bar{y}(x)) \equiv 0 \bmod (x)^{\beta}$ for $\beta=m$ but not $\beta=m+1$. Thus we have $\bar{y}(x)=a_{c} x^{c}+\ldots$ with $a_{c} \neq 0$. Now $f\left(x, x^{c} y\right)=$ $\sum a_{i j} y^{j} x^{\lambda}+$ higher terms (where the sum is taken over all pairs $(i, j)$ for which $a_{i j} \neq 0$ and $i+c j=\lambda_{c}$ is a minimum). If there is only one such term then, since $\lambda_{c}$ is minimal, the line $x+c y=\lambda_{c}$ must lie on only one side of the Newton Polygon, so $\lambda_{c} \leqslant m$. Since $a_{c} \neq 0$ we cannot have $f(x, \bar{y}(x)) \equiv 0 \bmod$ $(x)^{\lambda+1}$. However we have assumed $f(x, \bar{y}(x)) \equiv 0 \bmod (x)^{\beta}$ for $\beta \geqslant m+1$ so this case does not occur. There must, therefore, be two pairs $(i, j)$ in this sum. The line $x+c y=\lambda_{c}$ then coincides with a segment of the Newton Polygon with integral declivity and so there are exactly two terms in the sum. We write

$$
f\left(x, x^{c} y\right)=x^{\lambda} f_{1}(x, y),
$$

and note that $f_{1}(0, y)=\sum a_{i j} y^{j}$. Since $\beta \geqslant m+1 \geqslant \lambda_{c}+1$, $a_{c}$ must be a (nonzero) root of $f_{1}(0, y)=0$. Since there are only two terms in $f_{1}(0, y)$ any nonzero root is simple so $\partial f_{1}\left(0, a_{c}\right) / \partial y \neq 0$. Now clearly $f_{1}$ is the same as the 
$f_{1}$ obtained by applying the method of $\$ 2$ with $a_{0}=\cdots=a_{c-1}=0$ and $\lambda_{c}=r_{0}+\cdots+r_{c-1}$. At this stage we are now in the IFT situation and obtain a nonterminating branch in the tree diagram. The analysis of the preceding section shows that $\beta(\alpha)=\lambda_{c}-c+\alpha$ works on this branch. This completes the proof.

Simplicity of the Newton Polygon can be immediately detected. The proof of Theorem 2, however, applies in a more general situation. It should be observed that simplicity was used only to guarantee that $f_{1}(0, y)$ had no nonzero multiple root.

Let $\left(i_{1}, j_{1}\right), \ldots,\left(i_{k}, j_{k}\right)$ be the vertices of a segment of the Newton Polygon. We call $F(y)=\Sigma_{\nu} a_{i, j_{v}} y^{j_{v}}$ the associated polynomial of this segment.

THEOREM 3. If, for every segment with integral declivity, the associated polynomial has no nonzero multiple roots then the conclusion of Theorem 2 applies.

Example 6. $f(x, y)=y^{3}+x y^{2}-2 x^{2} y+x^{5}$ has a Newton Polygon identical to that in Figure 2. The associated polynomial for the initial segment is $F(y)=y^{3}+y^{2}-2 y$ which has no multiple roots. There are only two vertices on the other segment (which also has integral declivity). The two values of $\lambda$ are $\lambda_{1}=3$ and $\lambda_{3}=5$. Theorem 3 applies. $\beta=\max (3-1+\alpha, 5-3+$ $\alpha)=2+\alpha$.

By contrast, however, Theorem 3 does not apply to Examples 1 or 2 .

Proposition 5. If $A(x) \in k[x]$ and $g(x, y)=f(x, A(x)+y)$ then the same $\beta$ works for both $f$ and $g$.

Proof. Obvious.

In some circumstances we can easily convert a nonsimple Newton Polygon into a simple one by applying a transformation of this type. If $F(y)$ is the associated polynomial to a segment with integral declivity $d$ and if $a$ is a nonzero multiple root of $F(y)$ then $A(x)=a x^{d}$ will at least eliminate the problem with this root.

EXAMPLE. For Example 1 the associated polynomial for the segment of declivity 1 is $F(y)=-y^{2}-2 y-1=-(y+1)^{2} \cdot a=-1$ is a nonzero multiple root. Let $g(x, y)=f(x, y-x)$. Then

$$
g(x, y)=y^{5}-3 x y^{4}+2 x^{2} y^{3}+\left(2 x^{3}-x\right) y^{2}-3 x^{4} y+\left(x^{5}+x^{4}\right) .
$$

The Newton Polygon for $g(x, y)$ is 


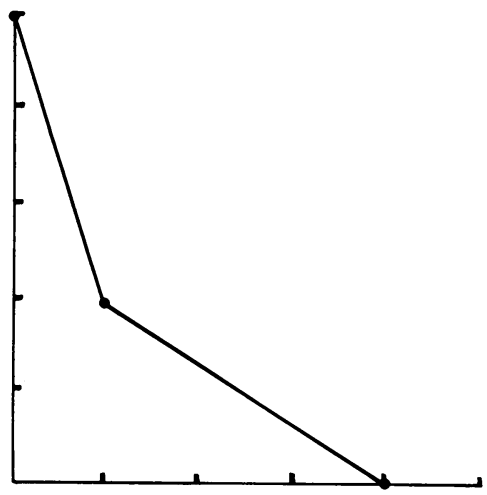

Figure 5

This Newton Polygon has no segment with integral declivity. Therefore $\beta=4+1=5$ satisfies the condition of Theorem I for $g(x, y)$ and so also for $f(x, y)$.

The same transformation also works for Example 2.

Now suppose $f(x, y)=y g(x, y)$ and that Theorem 2 or 3 applies to $g(x, y)$. Let $m$ and $\lambda_{d}$ be as in Theorem 2 for $g(x, y)$.

THEOREM 4. $\beta=m+\alpha$ satisfies the conditions of Theorem I for $f(x, y)$.

Proof. If $\bar{y}(x)$ satisfies $f(x, \bar{y}(x)) \equiv 0 \bmod (x)^{\beta}$ then either $\bar{y}(x) \equiv 0 \bmod$ $(x)^{\alpha}$ (in which case we are done) or else ord $(\bar{y}(x))=c<\alpha$.

$$
f(x, \bar{y}(x))=\bar{y}(x) g(x, \bar{y}(x)) \text {, }
$$

so

$$
g(x, \bar{y}(x)) \equiv 0 \bmod (x)^{\beta-c} .
$$

We have $\beta-c \geqslant m+\alpha-c \geqslant m+1$ so $\bar{y}(x)$ coincides mod $(x)^{\alpha}$ with a nonterminating branch for $g(x, y)=0$ provided $\beta-c>\lambda_{c}-c+\alpha$. Since $\lambda_{c} \leqslant m$ this is the case.

\section{BIBLIOGRAPHY}

1. M. Artin, Algebraic approximation of structures over complete local rings, Inst. Hautes Études Sci. Publ. Math. 36 (1969), 23-58.

2. J. L. Coolidge, $A$ treatise on algebraic plane curves, Dover, New York, 1959

3. W. Fulton, Algebraic curves, Benjamin, New York, 1969.

4. H. T. Kung and J. F. Traub, All algebraic functions can be computed fast, J. Assoc. Comput. Mach. (to appear).

5. J. C. Tougeron, Ideaux de fonctions differentiables, Springer, New York, 1972.

6. R. J. Walker, Algebraic curves, Princton Univ. Press, Princeton, N. J., 1950.

7. J. J. Wavrik, A theorem on solutions of analytic equations with applications to deformations of complex structures, Math. Ann. 216 (1975), 127-142.

Department of Mathematics, University of California at San Diego, la Jolla, CaliFORNIA 92093 